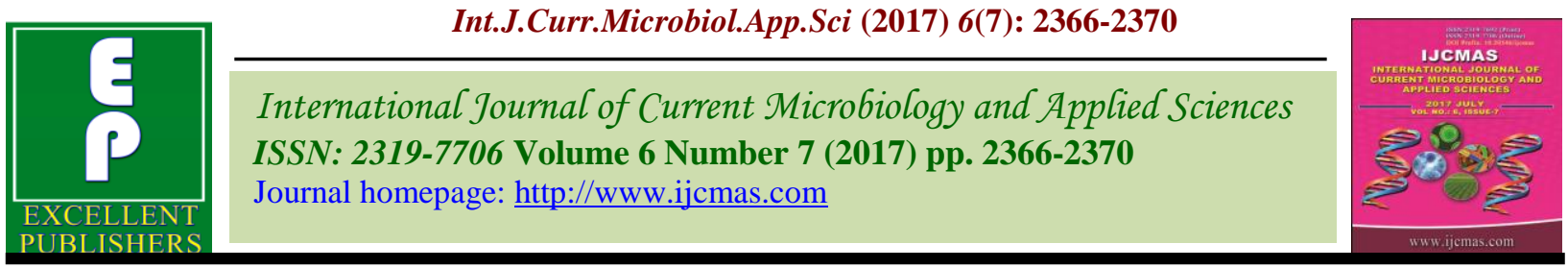

Original Research Article https://doi.org/10.20546/ijcmas.2017.607.338

\title{
Microbial Profile of High Vaginal Swab from Women of Reproductive Age Group in a Tertiary Care Hospital
}

\author{
Anna Cherian* and O. Sasikumari \\ Department of Microbiology, Govt. Medical College, Trivandrum, Kerala, India \\ *Corresponding author
}

\begin{abstract}
A B S T R A C T
Bacterial vaginosis, candidiasis and trichomoniasis, along with sexually transmitted infections are the common vaginal infections in women of reproductive age group in developing countries. Here the normal vaginal flora, consisting of peroxide-producing lactobacilli, are replaced by Gardnerella vaginalis, Mycoplasma hominis, Mobiluncus species, and anaerobic gram-negative rods. ${ }^{1}$ In the pregnant state microbial infections can lead to premature rupture of membranes which in turn can cause infant morbidity and mortality due to infections. Early detection and appropriate antimicrobial therapy can be life-saving by preventing vertical transmission of the pathogens. To find out the Microbiological profile of high vaginal swabs (HVS) from patients with various lower genital tract symptoms in the reproductive age group, attending the Obstetrics and Gynaecology Department at Sree Avittam Thirunal Hospital (SATH), Thiruvananthapuram, Kerala, South India. A hospital based study was done on 1315 high vaginal swabs collected during a period of two years, from May 2015 to April 2017.The samples were cultured aerobically on Blood agar (BA), Chocolate agar(CA)and Mac Conkey agar(MA) for microbial isolation. The overall positivity for pathogenic aerobic microbes was 234 (18\%), while 1081 (82\%) grew normal vaginal flora. Candida was the predominant pathogen in $125(53 \%)$ patients. In this study there was a significantly high rate of detection of Candida species when compared to bacterial isolates. This justifies the practice of empirical antifungal therapy without taking high vaginal swabs in patients with complaints of vaginal discharge or related symptoms especially in the Gynaecology OPD. The detection of pathogens in HVS culture and its appropriate treatment in symptomatic pregnant women is also necessary for a full term normal pregnancy, child birth and a healthy baby.
\end{abstract}

\section{Introduction}

The normal vaginal $\mathrm{pH}$ in women of reproductive age group is acidic $(\leq 4.5)$. This usually protects the vagina from the common pathogenic infections. Lactobacillus is a commensal in the vagina in abundant numbers. This bacillus maintains the acidic $\mathrm{pH}$ in the vagina by the production of lactic acid. The proportion of Lactobacillus, Mobiluncus and Gardnerella vaginalis, is scored in standard tables and is called Nugents scoring system. High Nugent scores have been associated with increased risk of sexually transmitted infections, preterm birth and adverse perinatal outcomes such as postabortal sepsis, recurrent abortion, chorioamnionitis, postpartum endometritis and pelvic inflammatory disease. The bacterial vaginosis in a pregnant female 
doubles the risk of preterm delivery ${ }^{2}$. An estrogen deficient state in vagina and the immune compromised status due to diabetes or other associated vaginal factors can lead to growth of abnormal flora which may in turn lead to infections. Maximum number of women seeking treatment was young, sexually active females of age group 26-35 yrs. vaginal infections are a common gynaecological problem in the study area. The main predisposing factors for symptomatic vaginal infections are poor hygiene, low socioeconomic status, and early sexual activity. In bacterial vaginosis $\mathrm{pH}$ is elevated. In vaginal candidiasis, it is $\leq 4.5$. Causes of vaginal discharge include physiological, infective (e.g. bacterial vaginosis, candidiasis, trichomoniasis) and non-infective (foreign bodies, cervical ectopy and genital tract malignancy). ${ }^{3}$

Women of reproductive age group are carriers of various organisms like Group B Streptococci, Candida, Listeria, Escherichia coli $(E$. coli) etc. E. coli which has a strong association with preterm delivery and very low birth weight infants that can cause lifethreatening neonatal diseases such as sepsis, meningitis, and necrotizing enterocolitis. During passage through the birth canal or before delivery $E$. coli can invade the chorioamnion or amniotic fluid and can lead to complications. ${ }^{4}$

Group B Streptococci (GBS) colonization is an independent risk factor for chorioamnionitis, being highest among women with the heaviest degree of colonization. The GBS colonised in the genital tract can enter the amniotic fluid and its aspiration can lead to invasive disease in the new born. Perinatal transmission can also occur across intact membranes. GBS also increases the risk of preterm labour, low-birth weight infant, postnatal and post-operative infections. Hence early detection of the carrier state can be very helpful to the obstetrician and the pediatrician.

The aim of the study is find the prevalence of pathogenic microbes in HVS and to detect the carrier stage in patients attending Obstetrics and Gynaecology department with various complaints. Clinical syndromes of GBS disease in newborns include sepsis, meningitis, pneumonia, cellulitis, osteomyelitis, and septic arthritis. Bloodstream infections, with or without pneumonia, are the main manifestations of GBS disease in infancy and are evident in $89 \%$ of cases. Meningitis is less common, occurring in approximately $10 \%$ of neonatal infection. Neurologic sequelae associated with GBS infection are most profound among premature infants. ${ }^{5}$

Acinetobacter species have emerged as significant nosocomial pathogens, with Acinetobacter baumannii being the most frequently isolated species. They are resistant to most antibiotics and can be found in both hospitalized patients and the community. A. baumannii infections were identified with adverse pregnancy outcomes. The risk for premature delivery is high with fetal or neonatal demise. ${ }^{6}$

Women of reproductive age with vaginal discharge should have a high vaginal swab (HVS) cultured if (7, 8, 9 and 10)

Postnatal or post miscarriage

Vaginitis without discharge

Pre or post gynaecological surgery

Pre or post termination of pregnancy

Symptoms not characteristic of bacterial vaginosis. 
Within 3 weeks of intrauterine contraceptive insertion.

\section{Materials and Methods}

\section{Study design}

Descriptive study

\section{Study setting}

Sree Avittam Thirunal Hospital (SATH), Thiruvananthapuram

\section{Inclusion criteria}

Females of reproductive age group attending gynaecology department at SATH, Thiruvananthapuram.

Study period was a period of two years, from5/2015 to 4/ 2017.

\section{Procedure}

A total of 1315 females of reproductive age group were screened during the study period. The details of the patient were collected using a proforma and high vaginal swabs were collected after taking consent from the patients by the gynaecologist.

Sterile double swabs were used. One was used for direct microscopical examination and the other was used for culture on BA, MA and CA. Identification and speciation of the predominant isolates were done by conventional methods. Antibacterial and antifungal susceptibility testing was done by disc diffusion method as per CLSI guidelines.

\section{Results and Discussion}

All the Candida spp isolated were sensitive to Fluconazole, Itraconazole, Voriconazole and Amphotericin B. Antifungal resistance was not noted in any of the isolates. All of the group B haemolytic Streptococci were sensitive to Penicillin.

Among the isolates of Enterococci, $7 \%$ were resistant to betalactam antibiotics and had high level resistance to aminoglycosides but all of them were sensitive to Vancomycin and Linezolid.

Klebsiella species (4.5\%) were sensitive to all the antibiotics including betalactams, aminoglycosides, and quinolones and resistant to ampicillin. Of these $2 \%$ were resistant to all antibiotics.

Only $1.2 \%$ of $E$. coli was multidrug resistant, showing resistance to quinolones, betalactams and aminoglycosides.

The two isolates of Acinetobacter spp were fully resistant to all the drugs.

A total of $1315 \mathrm{HVS}$ samples from females of reproductive age group were studied during the period of two years.

In the present study pathogenic bacteria were found in 234 samples out of the total of 1315.

This constitutes about $18 \%$ of the total samples. As per studies conducted by Gopalkumar et al., (2015) the overall positivity for pathogenic aerobic microbes was $27 \%$ and that of Candida was $7.3 \%$.

Of the 125 Candida isolated, C. albicans was $28 \%$ and the rest of them were Non albicans Candida, $72 \%$.

In the study by Siti et al., the recovery rate of Candida spp from pregnant women was $17.2 \%$ with $C$. albicans being the most common species detected $(83.5 \%)$, followed by C. glabrata (16\%) 2015 (Table 1 and Fig. $1)$. 


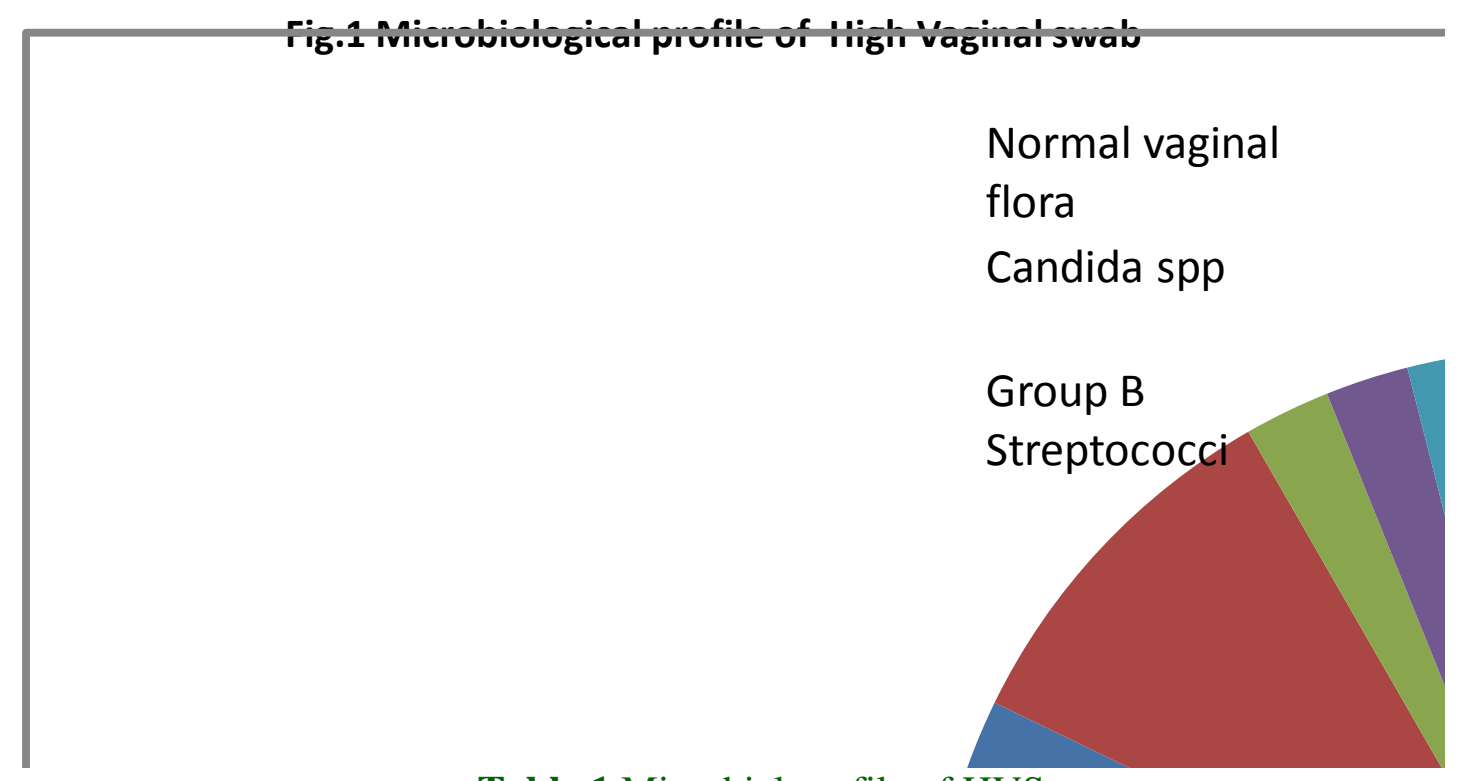

Table.1 Microbial profile of HVS

\begin{tabular}{|c|c|c|}
\hline S.no & Organism & Number (\%) \\
\hline 1 & Normal vaginal flora & 1081 \\
\hline 2 & Candida spp & 125 \\
\hline 3 & E. coli & 17 \\
\hline 4 & Klebsiella pneumoniae & 28 \\
\hline 5 & Staphylococcus aureus & 9 \\
\hline 6 & Group B Streptococcus & 29 \\
\hline 7 & Enterococcus spp & 2 \\
\hline 8 & Acinetobacter baumannii & $\mathbf{1 3 1 5}$ \\
\hline
\end{tabular}

The present study highlights the predominance of non albicans Candida in this particular geographical area. This justifies the role of empirical antifungal therapy by obstetricians and gynaecologists for patients with vaginal discharge. The next common isolate was Group B Streptococci, 29 (12\%). The carrier state of GBS was confirmed by rectal swabs of the patients. Yancey et al., in a study on peripartum infections, 216 women (26\%) were colonized with GBS. ${ }^{5}$ There were only two cases $(0.9 \%)$ of Acinetobacter spp. (Acinetobacter baumanni). Acinetobacter as such is not a vaginal pathogen but in these cases the isolates were obtained by repeated sampling. Both these cases gave history of $\mathrm{D} \& \mathrm{C}$ from local hospital which indicates that
Acinetobacter is an iatrogenic infection. A wide spectrum of antibiotics resistance (so called "pan-resistant") was seen with $A$. baumannii by Mai He et al., 6

In conclusion, Candida has been identified as the most common isolate among the pathogens from HVS. Hence the empirical use of antifungal is justified. The GBS forms $12 \%$ and E. coli, $7 \%$ of the total isolates which show the risk of neonatal infection in patients with premature rupture of membranes. Multi drug resistance in isolates indicates the importance of early culture and sensitivity for deciding empirical antibiotics in such patients, if they develop post natal uterine infections. 


\section{References}

1. Beverly E. Sha Hua Y. Chen Qiong J. Wang, M. Reza Zariffard Mardge $\mathrm{H}$. Cohen and Gregory T. Spear. Utility of Amsel Criteria, Nugent Score, and Quantitative PCR for Gardnerella vaginalis, Mycoplasma hominis, and Lactobacillus spp. for Diagnosis of Bacterial Vaginosis in Human Immunodeficiency Virus-Infected Women J Clin Microbiol. 2005 Sep; 43(9): $\quad$ 4607-4612.doi: 10.1128/JCM.43.9.4607-4612.2005

2. Linnéa Ekström, Annsofie Adolfsson, Henrik Ericson, Georgios Poutakidis, Georgios Charonis and Per-Göran Larsson Vaginal flora and urinary and vaginal group B Streptococci in early pregnancy.http://dx.doi.org/10.7243/205 2-6.

3. Gopal Kumar, Kalpana Singh Microbial Profile of High Vaginal Swab From Symptomatic Women of Reproductive Age Group:(2013): Volume 4 Issue 7, July 2015

4. Marijane A. Krohn, SoeSoeThwin, Lorna K. Rabe, Zane Brown, and Sharon L. Hillier. Vaginal Colonization by Escherichia coli as a Risk Factor for Very Low Birth Weight Delivery and Other Perinatal Complications The Journal of Infectious Diseases 1997;175:606-10

5. Anne Schuchat Epidemiology of Group B Streptococcal Disease in the United States: Shifting Paradigms. Clin.
Microbiol Rev. 1998 Jul; 11(3): 497513.

6. Mai $\mathrm{He}, \mathrm{MD}, \mathrm{PhD}$, Stefan Kostadinov, MD, FusunGundogan, MD, Judith Struminsky, MT(ASCP), MBA, Halit Pinar, MD, and C. James Sung, MDAJP Rep. 2013 May; 3(1): 51-56.Published online 2013 Feb 7. doi: 10.1055/s-00331334460

7. FFPRHC and BASHH Guidance. The management of women of reproductive age attending non-genito urinary medicine settings complaining of vaginal discharge. JFam Plan Reprod Health Care 2006; 32(1):33-41.

8. Caillouette JC, Sharp CF, Zimmerman J, Roy S. VaginalpH as a marker for bacterial pathogens and menopausal status. Am J Obstet Gynecol 1997; 176:1270-7.

9. UK national guidelines on sexually transmitted infections and closely related conditions. Sex Transm Infect 1999;7

10. Blackwell AL, Thomas PD, Wareham $\mathrm{K}$, Emery SJ. Health gains from screening for infection of the lower genital tract in women attending for termination of pregnancy. Lancet 1993; 342:206-10.

11. Siti Norbaya Masri, Sabariah Md Noor, Lailatul A kmar Mat Nor, Malina Osman, and MM Rahman Candida isolates from pregnant women and their antifungal susceptibility in a Malaysian tertiary-care hospital, Pak J Med Sci. 2015 May-Jun; 31(3): 658-661.

\section{How to cite this article:}

Anna Cherian and Sasikumari, O. 2017. Microbial Profile of High Vaginal Swab from Women of Reproductive Age Group in a Tertiary Care Hospital. Int.J.Curr.Microbiol.App.Sci. 6(7): 2366-2370. doi: https://doi.org/10.20546/ijcmas.2017.607.338 